Théologiques

Théologiques

\title{
Magie, superstition et tradition chrétienne : Pistes d'interprétation théologique
}

\section{Sophie Tremblay}

Volume 8, numéro 1, printemps 2000

La superstition

URI : https://id.erudit.org/iderudit/005029ar

DOI : https://doi.org/10.7202/005029ar

Aller au sommaire du numéro

\section{Éditeur(s)}

Faculté de théologie de l'Université de Montréal

\section{ISSN}

1188-7109 (imprimé)

1492-1413 (numérique)

Découvrir la revue

Citer cet article

Tremblay, S. (2000). Magie, superstition et tradition chrétienne : Pistes d'interprétation théologique. Théologiques, 8(1), 67-84.

https://doi.org/10.7202/005029ar
Résumé de l'article

La popularité croissante de pratiques et de croyances irrationnelles, endedans comme en dehors des grandes Églises, lance un défi important à lathéologie, celui d'examiner à nouveaux frais les phénomènes habituellementqualifiés de magie et de superstition. Les balises posées par lathéologie scolastique dans le domaine du merveilleux ont été remises enquestion radicalement par le choc de la modernité. Quelles pistes seraientalors susceptibles de renouveler l'interprétation théologique chrétienne dela magie et des phénomènes extraordinaires? L'article en explore deux : lerapport aux médiations dans le christianisme et l'espérance commerésistance à la fatalité. 


\section{Magie, superstition et tradition chrétienne :}

\section{Pistes d'interprétation théologique}

Sophie TREMBLay

Institut de pastorale

Montréal

Dans l'atmosphère de quête de sens et d'incertitude qui caractérise le temps présent, certains courants culturels ramènent sur la scène publique des pratiques et des croyances que la modernité avait marginalisées au point, croyait-on, d'en précipiter la disparition. En dépit des protestations des hommes de science, la «nébuleuse mystiqueésotérique ${ }^{1}$ jouit d'une importance sociale croissante en Occident. Ce courant aux contours flous, qui s'inspire des grandes religions orientales et de syncrétismes ésotériques nouveaux ou anciens, popularise les arts divinatoires, qu'il s'agisse du tarot, de l'astrologie, de la chiromancie, de la voyance, du Yi-King. Une pléthore de nouvelles pratiques de guérison (Reiki, polarité, toucher thérapeutique, utilisation des cristaux, etc.) s'inspirent explicitement de traditions préscientifiques auréolées de sacré. De même, on peut trouver facilement des ouvrages comme le traité de magie blanche des Wicca sur les rayons de n'importe laquelle librairie.

Le christianisme n'échappe pas au déferlement de cette vague. Une proportion importante des membres des grandes Églises fréquentent ces nouveaux lieux du spirituel sans y voir de contradiction avec la foi chrétienne. Par ailleurs, la religion populaire catholique, bastion du merveilleux, ne manque pas d'adeptes malgré le doute semé à son égard : les sanctuaires et lieux de pèlerinage demeurent largement fré-

1. La sociologue Françoise Champion utilise habituellement cette expression pour désigner le foisonnement de la religiosité parallèle. 
quentés. Et que dire de ces croyants atteints par une véritable fringale de miracles et d'apparitions...

À l'aune de la rationalité héritée des Lumières, toutes ces pratiques et croyances entrent dans la catégorie douteuse de la magie et de la superstition, ce qui devrait provoquer leur rejet. Et pourtant, elles jouissent d'une popularité croissante, que ce soit dans les nouveaux espaces du spirituel ou dans les religions établies. Ces faits lancent un défi important à la théologie, celui d'examiner à nouveaux frais les phénomènes habituellement qualifiés de magie et de superstition, ou du moins d'accepter de reposer la question. Dans cet esprit, nous nous intéresserons en premier lieu aux balises posées par la théologie traditionnelle dans le domaine du merveilleux. Ces balises sont toujours manifestes dans les définitions courantes de quatre termes importants pour notre propos : magie, superstition, miracle et prodige. Nous observerons aussi comment le choc de la modernité a bouleversé ces repères anciens. Dans un second temps, nous explorerons deux pistes susceptibles de renouveler l'interprétation théologique chrétienne de la magie et des phénomènes extraordinaires : le rapport aux médiations et l'espérance comme résistance à la fatalité.

\section{La théologie chrétienne aux prises avec la magie}

\subsection{Les balises traditionnelles du domaine du merveilleux}

La magie n'a jamais eu bonne presse en théologie chrétienne. Le christianisme a hérité de la condamnation de la magie dans les Écritures juives, mais de plus, la société romaine la redoutait. Les chrétiens des premiers siècles durent eux-mêmes se défendre face à l'accusation de pratiquer la magie. Cependant, c'est la scolastique qui semble avoir conféré à la théologie chrétienne de la magie ses formes classiques. Cette théologie propose des balises pour se repérer dans le domaine du merveilleux. Ces balises ont contribué à cristalliser dans notre culture les définitions mêmes des termes de magie, de superstition, de prodige et de miracle, telles qu'on les trouve aujourd'hui dans les dictionnaires et les encyclopédies de consultation courante.

La théologie chrétienne traditionnelle n'est prête en aucune façon à renier le merveilleux, même si elle rejette la magie. Elle ne fait qu'introduire une frontière en son centre. Le domaine du merveilleux se divise ainsi en deux champs antagonistes. D'une part, les prodiges 
et les miracles sont habituellement définis comme des événements extraordinaires qui paraissent défier le cours habituel des choses et où il est possible de surprendre Dieu en flagrant délit d'intervention. Le champ sémantique du terme "prodige » se développe surtout autour du caractère surprenant et inexplicable de l'événement. Dans le cas du " miracle », on insiste plutôt sur l'origine divine de l'événement et son caractère de révélation.

Le miracle est toujours attribué à Dieu, parfois grâce à l'action particulière d'un intercesseur, saint ou martyr, qui joue ainsi le rôle de catalyseur des faveurs divines. Les phénomènes extraordinaires attribués à l'action directe de Dieu deviennent des arguments de choix pour l'apologétique, des preuves pour appuyer la défense du christianisme et confondre ses adversaires. Puisqu'on considère Dieu luimême comme l'origine de ces événements, on leur donne le qualificatif de surnaturel. Enfin, on prétend également distinguer le phénomène surnaturel de la magie par ses fins, par sa signification morale et religieuse.

Dans le champ opposé, on retrouve la magie, qui serait une forme d'art mystérieux dont les procédés et les pratiques sont supposés opérer et produire eux aussi des phénomènes extraordinaires. Ces phénomènes peuvent éventuellement ressembler aux miracles de manière confondante, mais la théologie traditionnelle leur attribue une origine non divine : esprits, forces supra-humaines, démons, Satan, etc. À titre d'exemple, une guérison survenue pendant un temps de prière dans un sanctuaire marial pourrait être considérée comme un miracle. Mais si cette même guérison survenait dans le bureau d'un médium, la théologie traditionnelle la qualifierait de magie.

L'efficacité possible des rites magiques n'est pas d'ordre surnaturel puisqu'elle ne provient pas de Dieu. On la qualifie de préternaturelle. Ce terme ancien, dont le champ sémantique est mal délimité, désigne des dons dépassant les possibilités humaines habituelles, c'està-dire une forme de surnaturel non divin. On imaginait qu'Adam et Ève possédaient des dons préternaturels (appelés aussi justice originelle $^{2}$ ) qu'ils avaient toutefois perdus après la chute : exemption

2. À titre d'exemple, voir Louis OTT, Précis de théologie dogmatique, Mulhouse, Éditions Salvator, 1954, p. 153-155. 
de la souffrance et de la mort corporelle, connaissance infusée par Dieu, intégrité du corps et de la volonté.

En outre, on associe à une action supposée magique des fins plus égoïstes et individualistes : contrôler les forces de la nature à son avantage, influencer le cours des événements en se conciliant les forces qui les dirigent. Les prodiges magiques « manquent le plus ordinairement du caractère de dignité, d'utilité et de moralité qui distingue les miracles authentiques ${ }^{3}$ »; leur prestige est " d'ordinaire inutile à une fin supérieure, malfaisant même et malsain ${ }^{4}$ ». Ce jugement sur l'origine et les fins demeure le même, qu'on parle de magie blanche, de sorcellerie, de divination ou de simple superstition.

Dans son article du Dictionnaire de théologie catholique de 1927, L. Gardette définit la magie comme une espèce de superstition. Sous l'influence de Thomas d'Aquin, il considère la superstition comme un péché contre la vertu morale de religion, un vice qui lui est opposé. La superstition, et donc la magie, peut être comprise comme un culte rendu à un autre qu'à Dieu (culte que l'Ancien Testament dénonce comme idolâtre) ou encore un culte rendu à Dieu d'une manière qui lui déplaît ou lui fait injure. Les rites ne sont efficaces que si Dieu en a décrété ainsi, affirme Gardette. Il y aurait superstition à attendre de telle prière ou de telle coutume une efficacité que Dieu n'y aurait pas mise. La définition du dictionnaire Larousse demeure dans le même ordre d'idée en définissant la superstition comme une "déviation du sentiment religieux, fondée sur la crainte et l'ignorance».

La théologie traditionnelle de la magie se fonde donc sur l'idée de révélation pour ranger l'efficacité des sacrements du côté du surnaturel : "Si un rite sacré est révélé ou considéré comme révélé, si son efficacité lui vient de la volonté divine, il faut le rattacher non à la magie, mais à la religion. " 5 Toutefois, ceci n'exclut pas la possibilité qu'ils soient pratiqués de manière superstitieuse. Bref, il y a superstition à attendre des résultats merveilleux de moyens disproportionnés. Plus le phénomène attendu est extraordinaire, plus il risque de s'agir

3. H. LECLERCQ, " Magie » dans Dictionnaire d'archéologie chrétienne et de liturgie, tome X, partie 1, Paris, Letouzey et Ané, 1931, col. 1068.

4. L. GARDETTE, "Magie » dans Dictionnaire de théologie catholique, tome IX, partie 2, Paris, Letouzey et Ané, 1927, col. 1514.

5. Ibid., col. 1513. 
de magie. Mais l'invocation de puissances autres que celle de Dieu est le facteur le plus décisif, qui permet à la théologie traditionnelle de trancher sans équivoque.

Néanmoins, ces affirmations pleines d'assurance sur l'origine et la fin des phénomènes extraordinaires ne fournissent aucune réponse à une question qu'elles présupposent pourtant : quelle est la nature véritable de la magie? "Sous cette épithète magique, nous ne savons pas beaucoup mieux ce qu'il faut grouper que ce qu'il faut exclure ", écrit H. Leclercq. ${ }^{6}$ Et Gardette de renchérir : "S'il est difficile de bien dégager la notion de magie des notions plus ou moins voisines, de lui attribuer toute sa compréhension, rien que sa compréhension, il est incomparablement plus malaisé de se prononcer sur la réalité de la magie. $"{ }^{7}$ Ces remarques font ressortir la fragilité des définitions invoquées, et la nécessité de les garder ouvertes.

\subsection{Le choc de la modernité}

Le rationalisme moderne pose sur la magie un regard totalement différent de celui de la théologie traditionnelle. Pendant les derniers siècles du Moyen Âge, on avait toléré la cohabitation entre la rationalité de la dialectique scolastique et les pratiques populaires mélangeant allègrement des survivances païennes au christianisme. Cette tolérance s'est évanouie au moment où la modernité prenait son essor, au tournant des XVIIle et XVIIIe siècles. L'élimination de toute superstition faisait partie du programme des Lumières. Comme le catholicisme était devenu une source particulièrement riche de croyances irrationnelles, il a subi la foudre de la critique, par exemple dans l'Encyclopédie et dans les écrits de Voltaire et de Diderot. Soulignons en outre que les grands réformateurs protestants se sont attaqués avec virulence aux coutumes et aux rites catholiques (dont les sacrements, hormis le baptême et l'eucharistie) qui leur semblaient infidèles aux Écritures parce que porteurs de magie et de superstition. Dans le même ordre d'idée, l'instruction religieuse et l'alphabétisation constituaient le fer de lance de la réforme catholique. "La Réforme, catholique ou protestante, n'est autre chose que la christianisation systématique d'une masse encore en partie

6. H. LECLERCQ, loc. cit., col. 1067.

7. L. GARDETTE, loc. cit., col. 1515. 
païenne, engluée dans des superstitions païennes ou dans un christianisme grossier ou paganisé. ${ }^{8}$

Tandis que le christianisme esthétique et romantique du XIXe siècle récupérait quelques pratiques anciennes, les sciences et les techniques se développaient. À la fin du XIXe siècle et au début du XXe siècle, les pionniers des sciences humaines se donnaient pour mission de comprendre les phénomènes humains d'un point de vue aussi objectif et rationnel que possible, de manière à pouvoir expliquer les comportements collectifs et le fonctionnement des sociétés humaines comme on tente d'expliquer des phénomènes astronomiques ou géologiques. La magie et la religion ont bien sûr attiré l'attention des anthropologues, ethnologues et sociologues, qui pouvaient établir des comparaisons entre l'Occident et plusieurs cultures non occidentales. La psychanalyse freudienne s'est également intéressée à la question de la pensée magique. Les théories de Frazer, de Durkheim, de Mauss et Hubert, et plus tard de Lévi-Strauss ont obligé la théologie à reconnaître au moins la complexité de la question. Mais en cherchant à expliquer la nature et l'origine de la magie, les sciences humaines devaient aussi théoriser sa relation avec la religion. L'entreprise devenait alors plus menaçante pour les théologiens, qui s'y sont intéressés tout en opposant pendant longtemps une forte résistance.

Les sciences humaines ont remis en question la validité de la théologie traditionnelle de la magie parce que cette dernière admettait d'emblée l'existence du merveilleux et d'un arrière-monde. Certains phénomènes merveilleux lui servaient même de preuves spécifiques des réalités religieuses proposées à l'adhésion des croyants. Les sciences humaines cherchent plutôt à démonter les mécanismes sociaux, culturels et psychologiques qui construisent l'effet de merveilleux. La distinction entre magie et miracle perd toute pertinence dans cette opération qui se veut démystificatrice et qui rejette toute idée de transcendance. On propose ainsi des hypothèses rationnelles pour expliquer tout phénomène extraordinaire, quelle qu'en soit l'origine. Ces hypothèses peuvent diverger entre elles mais s'accordent au moins pour enlever toute crédibilité à l'ancienne théologie. Cette dernière est maintenant disqualifiée dans l'opinion publique en Occident, sauf

8. Philippe ARIÈS, "Religion populaire et réformes religieuses " dans $L a$ Maison-Dieu, 122 (1975), p. 86. 
dans certains groupes religieux en rupture avec le courant dominant de la culture.

Depuis le début du XXe siècle, plusieurs théories ont été formulées sur l'origine de la magie et de la religion. Aucune ne fait l'unanimité. De toute façon, nos connaissances limitées des origines des cultures humaines ne permettent pas de trancher un tel débat. Cependant, les sciences humaines mettent en lumière la coexistence habituelle de la magie et de la religion. Tantôt en concurrence, tantôt en compromis, elles sont mêlées l'une à l'autre de telle manière qu'il est difficile de faire passer une frontière nette entre les deux. De plus, on peut parfois noter la contagion de l'une sur l'autre. Ainsi, bien que l'on se soit acharné contre la magie dans l'histoire du christianisme, les sciences humaines nous rappellent que l'expérience magique constitue un fait de notre propre culture, et pas seulement un trait exotique de civilisations prétendument plus primitives que la nôtre. Au fond, la modernité n'a-t-elle pas repris avec plus de radicalisme la lutte contre la magie héritée des origines du christianisme?

Aujourd'hui, la magie n'est pas un sujet courant en théologie. Seuls les théologiens les plus conservateurs n'ont pas rejeté la position traditionnelle. L'esprit critique hérité de la modernité a généré une méfiance systématique à l'égard du merveilleux et des arrière-mondes, méfiance qui définit les limites de la crédibilité des discours. Ce trait a marqué plusieurs courants théologiques importants du XXe siècle (pensons à Bultmann, Bonhoeffer, Tillich), mais aussi la sensibilité des croyants ordinaires. C'était le fond de l'inquiétude de John A . T. Robinson dans les années soixante :

Nous ne pourrons finalement pas plus convaincre les hommes de l'existence d'un Dieu "au-delà ", appelé à ordonner leur existence, que les persuader de prendre au sérieux les dieux de l'Olympe. [...] Nous sommes appelés à beaucoup plus qu'une simple réaffirmation, en termes modernes, de l'orthodoxie chrétienne. Un remodelage beaucoup plus radical est à mon avis nécessaire et, dans ce processus, les catégories les plus fondamentales de notre théologie, de Dieu, du surnaturel et de la religion elle-même, doivent être de nouveau jetées au creuset.?

9. John A. T. ROBINSON, Dieu sans Dieu, Paris, Nouvelles Éditions Latines, 1964 , p. 14 et 58-59. 
Si les phénomènes extraordinaires apportaient autrefois une crédibilité au discours chrétien, il n'en est plus de même aujourd'hui. Combien de croyants avouent croire malgré eux! On n'établit plus de rapport de cause à effet entre un miracle et le monde surnaturel, parce qu'on conçoit mal comment Dieu pourrait agir directement comme cause première d'un phénomène extraordinaire, à l'encontre des lois naturelles (ce qui était le cœur de la définition thomiste du miracle). On peut reconnaître ici l'héritage de philosophes comme Spinoza, Bayle et Hume. L'ambiguité du merveilleux, tant religieux que magique, sème le malaise, même dans le monde de la théologie : « on ne sait comment l'expliquer, [et ] l'on croit qu'un jour la science pourra, sinon avoir le dernier mot, fournir des éclaircissements à propos du fait miraculeux ». ${ }^{10}$

Nombre de théologiens et de croyants ont accueilli la critique moderne comme une libération, comme une possibilité d'être enfin " honnêtes avec Dieu », suivant l'expression de Robinson. Dès lors, les pratiques chrétiennes traditionnelles tendent à devenir difficiles à tolérer. Alors que la théologie traditionnelle situait la magie en extériorité tout en conservant en elle une place pour les phénomènes extraordinaires, on en est venu à subodorer de la magie ou de la superstition partout, et à tenter de l'expurger le plus possible des pratiques chrétiennes, en commençant par la religion populaire, les sacrements et la pratique religieuse saisonnière.

Bien que cette révolution n'ait pas encore atteint l'ensemble des baptisés et des croyants, on peut reconnaître les bienfaits de cette ouverture à la rationalité moderne survenant après une longue période de résistance opiniâtre. Néanmoins, la persistance des pratiques qu'on a tenté de faire disparaître et l'influence actuelle de la " nébuleuse mystique-ésotérique » posent des questions dérangeantes à la théologie. En dépit de son intérêt, la vision rationaliste de la religion et de la magie est-elle complète? Se pourrait-il qu'elle mutile l'expérience humaine de l'une de ses dimensions? La théologie peutelle résister au chant des sirènes du positivisme scientifique sans se complaire paresseusement dans l'irrationnel? Se laissera-t-elle interpeller par la crise de la modernité? Bref, est-il possible de porter un regard critique sur la magie et la superstition sans sacrifier le mystère?

10. Gérard ROCHAIS, "Dieu fait-il des miracles? " dans Prêtre et Pasteur, décembre 1988, p. 673. 


\section{Deux pistes actuelles d'interprétation théologique}

\subsection{Le rapport aux médiations dans le christianisme}

L'attrait actuel pour la divination, les médecines parallèles, la voyance, l'intuition pure, tant dans les Églises qu'en dehors d'elles, vient rappeler aux chrétiens que leur tradition établit une relation dialectique entre deux modes de pensée, le mythos et le logos. Mythos et logos ont tous deux façonné l'histoire du christianisme et l'histoire de l'Occident. Le logos, c'est l'univers de la rationalité, du concept, du raisonnement, de la preuve, de la démonstration. Il s'adresse à la dimension intellectuelle de l'expérience humaine. La modernité a valorisé le logos au point de le déifier (rappelons-nous la Déesse Raison de la Révolution française). Depuis la scolastique du Moyen Âge, la théologie chrétienne s'est située en plein cœur de ce mouvement de valorisation de la raison, dont elle a d'ailleurs cependant perdu l'initiative. Au XXe siècle, il lui a fallu rattraper le retard accumulé pendant quelques siècles de résistance. Mais de toute manière, l'exercice du logos a toujours fait partie de l'expérience historique du christianisme, surtout en raison de son héritage culturel gréco-romain.

Le mythos, qui constitue l'autre pôle de cette relation dialectique, se nourrit plutôt de symboles, de métaphores, d'images. Il correspond à la manière dont nous tentons d'habiter le monde, d'entrer en communion avec le cosmos et avec les autres, d'une manière qui engage toute la personne en quête de sens. Le mythos provient de notre héritage juif, mais il était également présent dans l'ensemble du monde antique. Depuis les Lumières, le mythos a perdu graduellement son importance en Occident. On l'a associé aux enfants et aux peuples primitifs, ce qui traduit bien le mépris dans lequel on a tendance à le tenir. Cependant, la contre-culture d'où est issue la « nébuleuse mystique-ésotérique » a opéré un retour débridé au mythos dans une perspective d'opposition à la victoire sociale du logos.

Dans le christianisme, ces deux modes de pensée entrent traditionnellement en rapport dialectique, en tension dynamique. Si le mythos prend toute la place, la possibilité de prendre un recul critique face aux discours et aux pratiques disparaît. La perspective historique s'estompe dans la fascination de l'origine. La relation au monde, aux autres, au cosmos, glisse vers la fusion. Mais d'autre part, la prédominance absolue du logos devient vite appauvrissante et dévitalisante. 
Elle tend à éliminer l'engagement, la subjectivité et la quête de sens en privilégiant une position d'extériorité par rapport au monde, à soi et aux autres.

De ce rapport dialectique, on pourrait déduire que la théologie traditionnelle de la magie et du miracle s'enracine surtout dans le mythos, mais en le prenant au pied de la lettre, sans recul suffisant. La critique moderne se situe globalement du côté du logos, de manière à évacuer le mythe et le symbole. Ces positions demeurent donc trop partielles et trop fragiles. Mais une position équilibrée devrait tenir compte des deux, en particulier pour éviter de renier les avancées permises par la modernité.

Même si nous faisons reculer chaque jour les frontières de notre ignorance, la question du sens continue de se poser pour les individus et les collectivités avec urgence. En dépit des avancées toujours plus audacieuses de la technologie, de la circulation croissante d'informations et de connaissances, les humains continuent de lever des yeux interrogatifs sur cet univers immense où ils ne sont qu'un grain de poussière. S'ils portent le regard sur leur planète bleue, l'interrogation n'est pas moins profonde. L'histoire du XXe siècle à elle seule suffit à démontrer que, malgré leurs prétentions, les humains ne maîtrisent pas encore l'univers, ni la Terre, ni même leurs sociétés. Les enfants qui naissent au tournant du millénaire apprendront dans le regard de leurs parents et de leurs grands-parents que le monde peut être menaçant, qu'il n'est pas si facile à comprendre, que plusieurs sens différents et divergents peuvent lui être donnés, qu'il existe une multitude de manières de l'habiter. De plus, chaque jour se produisent des phénomènes intrigants résistant aux explications. Ainsi l'immanence de l'expérience quotidienne se laisse-t-elle parfois trouer de bribes d'une transcendance difficile à nommer, lente à apprivoiser. Une transcendance qui se présente moins comme un objet immense posé parmi les autres que comme un vide, une absence, un manque, une question lancinante. C'est ce que les chrétiens appellent mystère.

Le concept habituel du mystère dans le catholicisme fait appel à la même conception de la révélation que la théologie traditionnelle de la magie. Le mystère se réduit au contenu propositionnel d'un décret divin, face auquel la raison doit s'incliner même si ce contenu lui paraît obscur. La raison et le mystère, le naturel et le surnaturel se trouvent en compétition et coupent l'humain en deux. Nul besoin de 
préciser que cette conception n'a pas résisté au rationalisme moderne. Précisons également que la tension entre mythos et logos se transforme ici en une guerre stérile. Karl Rahner propose une manière plus riche de voir le mystère, comme une dimension fondamentale et irréductible de l'expérience humaine, celle de l'ouverture à la transcendance (l'abyssal, le vide, l'horizon infini) :

Nous sommes toujours déjà familiers avec le mystère. Nous l'aimons depuis toujours. Car rien n'est plus familier et plus intelligible par soi à l'esprit qui a pris conscience de soi que la question silencieuse qui s'élève au-dessus de tout ce qui est déjà conquis et dominé, que l'acceptation humble et amoureuse d'une question qui nous dépasse. ${ }^{11}$

Les traditions religieuses de l'humanité constituent des voies partagées pour apprivoiser ce mystère inhérent à l'existence, mystère à la fois passionnant et angoissant. Mais quelle serait l'originalité du christianisme à cet égard?

Dans son rapport au mystère, le christianisme confesse un Dieu transcendant présent dans l'immanence. ${ }^{12} \mathrm{Il}$ s'agit d'une position tout à fait particulière qui se traduit en tension dynamique. Pour l'Islam et, dans une moindre mesure, pour le judaïsme, la transcendance impose une distance absolue entraînant le rejet des images et une méfiance sans compromis à l'égard des médiations. Au contraire, dans les religions païennes de l'antiquité, les médiations immanentes (la royauté, les images, les rites) pouvaient être considérées comme une traduction directe de la transcendance, sans prise de distance. La foi au Christ ressuscité cristallise un rapport entre transcendance et immanence différent de ces deux positions. Le Christ ressuscité est présent, mais demeure insaisissable. Il ne peut être reconnu qu'à travers des médiations mais ne se confond jamais avec elles, comme l'illustre à merveille le récit des disciples d'Emmaüs (Lc 24, 13-35).

Nous pouvons donc constater l'importance de consentir aux médiations dans toute leur diversité. La tradition chrétienne en privilégie quelques-unes, mais il serait impossible de faire une liste exhaus-

11. Karl RAHNER, Écrits théologiques, Tome VIII, Paris, Desclée de Brouwer, 1967, p. 82-83.

12. Cette section du texte s'inspire des ouvrages de Louis-Marie CHAUVET en théologie sacramentaire. 
tive de toutes les médiations possibles : leur seule frontière est celle de l'expérience humaine. Pour cette raison, même si les phénomènes extraordinaires paraissent douteux, ils peuvent jouer le rôle de véritables médiations dans l'histoire de certaines personnes, de certains groupes. La fascination pour le merveilleux peut témoigner d'une ouverture nouvelle au mystère. Quelle que soit la nature ou l'origine de l'événement vécu, quelque explication que l'on en donne, c'est la signification revêtue par l'événement dans la trame d'une histoire de vie qui peut en faire une médiation significative. Ce sens ne sera pas évident aux yeux de tous ; mais pourquoi devrait-il l'être? Il ne s'agit pas de considérer comme signe un événement brut mais plutôt un événement interprété dans un cheminement, dans une histoire.

Toutefois, en vertu de ce rapport unique entre transcendance et immanence, consentir aux médiations signifie aussi demeurer conscient de leur finitude, de leur matérialité, de leurs limites. Si les médiations sont incontournables, on s'illusionnerait à les croire transparentes, à espérer lever totalement le voile du mystère pour capturer son essence dans les filets du désir. Les médiations nous font pressentir la levée du voile mais ne la réalisent jamais. Elles ne comblent jamais la brèche ni le manque. Elles ne nous permettent pas de faire l'économie de la distance et de l'altérité mais nous amènent à renoncer à toute mainmise sur Dieu, à " consentir à la présence du manque de Dieu ", comme l'exprime Louis-Marie Chauvet. Dans la fascination pour les phénomènes extraordinaires (magie, divination, miracles, apparitions, etc.), on peut aussi reconnaître cette tentation fusionnelle et captatrice, cet appétit de théophanies qui figent le désir en le fixant sur un seul objet. La division opérée par la théologie traditionnelle de la magie ne tient plus face à ce rappel exigeant. L'origine supposée des événements ne joue plus de rôle discriminatoire. Les manifestations extraordinaires attribuées à l'action directe de Dieu posent autant de problèmes que les autres puisqu'elles tombent dans le piège de l'absolutisation des médiations, du manque de recul à leur égard, de la chosification du mystère. À cet égard, les exhortations vigoureuses des prophètes de l'Ancien Testament continuent d'interpeller les chrétiens d'aujourd'hui à la vigilance contre l'idolâtrie.

Ainsi, le principe sacramentel qui oriente les rapports des chrétiens aux médiations pourrait être comparé à une ligne de crête : une voie escarpée entre le consentement aux médiations et le recul critique qui relance le désir et la quête. Le mépris des médiations constituerait 
une manière de dévier de cette ligne de crête, que l'on méprise toute médiation dans son principe ou simplement quelques médiations jugées déplaisantes, inconvenantes, inadéquates, ridicules. La volonté d'expurger tout ce qui peut ressembler de près ou de loin à la magie, à la superstition ou à la religion populaire, tombe dans ce travers. Mais l'absolutisation des médiations s'éloigne tout autant de la ligne de crête. Quelle que soit la voie par laquelle survient l'ouverture au mystère dans le parcours d'une personne ou d'un groupe, il serait dangereux de s'y installer confortablement dans l'immobilisme de la certitude et la conviction d'avoir "trouvé » Dieu.

\subsection{Magie, espérance et fatalité}

Si la survivance de pratiques magiques, superstitieuses ou divinatoires paraît si surprenante, on pourrait sans doute l'attribuer à l'échec d'un rationalisme triomphant qui avait annoncé avec pompe leur disparition certaine. En fait, ce type de pratique n'a-t-il pas toujours existé, quoique sous les formes les plus diverses, de l'antiquité à l'aube du XXIe siècle? Existe-t-il des sociétés ou des civilisations où l'on n'en aurait trouvé aucune trace, où personne n'aurait manifesté d'inquiétude au sujet de son destin au point d'espérer en violer les secrets ou se concilier les forces qui l'influencent?

La magie des sociétés pré-scientifiques cherche en partie à se concilier les forces de la nature : "faire tomber la pluie, éloigner la grêle, chasser les nuages, apaiser les vents, fertiliser le sol, multiplier les animaux $»{ }^{13}$ On peut imaginer sans peine comment James G. Frazer en a déduit que la pensée magique était une forme de pseudo-science ou de pseudo-art, un système logique mais non rationnel, une technique visant à manipuler la nature pour en obtenir des résultats précis. Aujourd'hui, la science offre des moyens toujours plus puissants d'expliquer et de contrôler ces forces. Pourtant, magie et divination croissent en popularité. Répondraient-elles à des besoins différents de ceux que la science et la technique peuvent combler si efficacement?

L'expérience magique ne se limite pas à une tentative de contrôler les forces de la nature. Elle a toujours constitué une médiation pour la traversée d'épreuves et d'étapes importantes dans l'existence indivi-

13. H. LECLERCQ, loc cit., col. 1069. 
duelle et la vie des groupes sociaux. La science ne saurait répondre à ce besoin de sens, de symbolisation, d'intégration, d'accompagnement. En tous temps et en tous lieux, les passages, les ruptures et la maladie ne demeurent-ils pas exigeants à vivre et à assumer? En outre, l'appréhension du malheur, de la malchance et des catastrophes n'est pas disparue dans nos sociétés même si l'espérance de vie continue de s'allonger. Qui ne craint les coups du sort : accidents graves, maladies mortelles, décès ou suicide d'un proche, perte d'emploi, rupture amoureuse, etc.? La quête inquiète du bonheur individuel contribue sans doute à aviver ces appréhensions.

De plus, l'équilibre social est fragile en Occident et garantit de moins en moins la protection des citoyens contre les « catastrophes collectives » que sont les mises à pied massives dans les entreprises, l'élargissement du fossé entre pauvres et riches, la précarité et la mobilité extrêmes, l'accès plus difficiles aux services essentiels, les complexes problèmes mondiaux d'économie, d'écologie et de droits humains qui paraissent insolubles. Les promesses des lendemains qui chantent n'ont pas été tenues et cette déception hypothèque la possibilité même d'espérer. Les discours utopiques semblent hors de propos aujourd'hui, alors qu'ils généraient encore l'enthousiasme vingt ans plus tôt. La question de l'avenir prend un tour particulièrement radical dans le contexte actuel. Les membres des plus jeunes générations, à qui pourtant l'avenir appartient, dit-on, gribouillent « No future » sur les murs des cités.

L'idée de destin et de fatalité semble sous-jacente à cette angoisse devant l'avenir proche ou lointain : la conviction que les événements survenant dans la trame des existences individuelles et collectives sont en quelque sorte " déjà écrits ", programmés, éventuellement prévisibles. Qui tisse le fil de ce destin? Quels dieux? Quelles forces cosmiques inscrites dans les étoiles? Ou quel Dieu provident, omniscient et tout-puissant? Ce destin est-il régi par une loi inflexible et aveugle de fatalité? En tentant de deviner les secrets de ce destin (grâce à l'astrologie, à la voyance, à la chiromancie, au channelling ou à d'autres techniques), doit-on simplement se préparer à faire face à ce qui ne peut être évité? Y a-t-il moyen de changer le cours de choses? Comment ce destin oriente-t-il la construction de l'identité individuelle et du rôle social de chacun? Comment conditionne-t-il les choix collectifs? La fascinante idée de fatalité, qui connote le déterminisme et la mort, génère un certain sentiment d'écrasement mais peut aussi cal- 
mer l'incertitude et l'insécurité face à un avenir énigmatique et menaçant : "L'amor fati reste une passion humaine, et l'antifatalisme est une rude et difficile protestation. Le fatalisme trouve en nous une étonnante complicité. Cette complicité n'est d'ailleurs pas inintelligible. ${ }^{14}$

Cependant, même si le christianisme n'a pas les mains blanches par rapport à la crise actuelle de la modernité, même s'il ne s'est pas toujours bien défendu contre l'idée de destin (en font foi les innombrables discussions sur le salut et la prédestination, la grâce et le libre arbitre), même si on peut lui reprocher d'avoir trop souvent prêché la résignation de manière révoltante, il demeure tout de même porteur d'une vision de la transcendance qui fonde l'autonomie et la liberté humaine, qui ouvre l'avenir sous le signe d'une espérance défatalisante. Au nom de l'espérance qui, écrivait Moltmann, est bien plus qu'un aspect isolé du christianisme, les croyants sont appelés à assumer leur liberté même si elle demeure limitée et conditionnée. Cette espérance ressemble davantage à un défi permanent qu'à un conte lénifiant et naïf sur des lendemains idylliques.

Qui espère en Christ ne peut plus s'accommoder de la réalité donnée, mais commence à en souffrir, à la contredire. [...] Si nous n'avions sous les yeux que ce que nous voyons, alors nous nous accommoderions, de gaieté de cœur ou à contrecœur, des choses telles qu'elles sont. Mais que nous ne nous accommodions pas, qu'entre nous et la réalité il ne s'établisse pas de sereine harmonie, c'est là que se produit l'espérance inextinguible. [...] Ouverte au monde par la promesse de Dieu donnée dans la Résurrection du Christ [...], cette espérance fait de la communauté chrétienne un foyer d'agitation constante, au sein des sociétés humaines qui veulent se stabiliser en " cité permanente» $(\mathrm{He} 13,14)$. Elle fait de la communauté la source d'où partent des impulsions toujours nouvelles à réaliser le droit, la liberté et l'humanité d'ici-bas, à la lumière de l'avenir annoncé qui doit venir. ${ }^{15}$

Cette espérance ne se prétend pas au-dessus de la souffrance. Elle passe par la croix, qui ne permet pas de minimiser la réalité de la souffrance et de la mort. Réalité qu'il serait facile de considérer comme une

14. Adolphe GESCHÉ, « Pour une anthropologie de destinée. Les chemins théologiques de la liberté » dans Adolphe Gesché (dir.), Destin, prédestination, destinée, Paris, Cerf, 1995, p. 144.

15. Jürgen MOLTMANN, Théologie de l'espérance, Paris, Cerf, 1970, p. 18. 
fatalité, comme un destin hideux et inéluctable, comme la rançon de la liberté, mais qui, acceptée et assumée dans l'amour, devient paradoxalement signe de salut et de libération. La résurrection du Crucifié, inédite, inattendue, scandaleuse, met en échec l'idée de destin et renverse la conception habituelle d'une vie réussie. Sans faire l'économie de la finitude, elle en transcende les limites. Elle propose non la résignation mais le courage de combattre librement jusqu'au bout. Cette espérance est un ferment de liberté, un projet audacieux marqué par l'excès.

Mais la liberté, même conditionnée, ne fait peut-être pas partie des souhaits spontanés des humains : "Tout épris qu'il soit de liberté, l'homme est-il en effet capable d'en supporter d'un bout à l'autre l'effort, de surmonter réellement tout obstacle? [Nombreux sont les comportements] qui témoignent, qu'on le regrette ou non, que la liberté peut être plus lourde à porter que la fatalité. »16 Au XIXe siècle, Toqueville, fin observateur de la démocratie en Amérique, pressentait qu'entre l'égalité et la liberté, les citoyens préféreraient la première même s'il leur fallait sacrifier la seconde. De même, le psychanalyste Erich Fromm constatait les effets pervers de la peur de la liberté sur l'histoire du XXe siècle : en dépit des idéaux modernes, cette peur a permis aux pires dictateurs de se donner un pouvoir immense et destructeur sur des sociétés fascinées et facilement soumises.

Enfin, en regardant l'histoire du christianisme, on peut constater que l'image d'un Dieu omniscient et tout-puissant, calquée sur le portrait d'un potentat tyrannique, a souvent conforté la peur de la liberté, la résignation et l'abandon à un destin tout tracé dans les cieux. Heureusement, l'histoire nous rappelle également qu'on trouve à toutes les époques des croyants et des communautés assez courageux pour défier cette pesanteur, pour assumer jusqu'au bout l'audace de l'espérance.

L'attrait pour la magie, la superstition, la divination, rend visible l'amor fati potentiellement présent dans le cœur de chaque croyant, de chaque communauté. Il indique à quel point cette pesanteur de destin qui nous habite tous nécessite un travail en profondeur en nous et autour de nous. Sinon, chasser les sorcières, dénoncer les pratiques et les croyances des " autres ", dans l'Église ou la société, ne demeure-til pas un moyen de déplacer à l'extérieur un combat avant tout inté-

16. Adolphe GESCHÉ, loc. cit., p. 145. 
rieur, un moyen de se dérober à un mouvement de conversion jamais terminé? Toutefois, ce combat met en évidence la nécessité de questionner et d'élaguer les images de Dieu véhiculées dans la prière, la liturgie, la catéchèse, la pastorale et la théologie. Plus encore, les croyants sont-ils prêts à assumer quotidiennement ce combat pour que leurs communautés demeurent ces «ferments d'agitation » dont parle Moltmann? Sinon, qu'auraient-ils encore de neuf à apporter au monde?

\section{Conclusion}

Ainsi, les crises profondes que traversent actuellement nos sociétés contribuent à rendre caduques les anciens discours théologiques sur la magie. Les distinctions claires mais fragiles de la théologie traditionnelle n'ont pas résisté au choc de la modernité. Si l'on tentait de les dépoussiérer pour les mettre au goût du jour, elles favoriseraient un retour en arrière plutôt qu'un dépassement du rationalisme moderne. C'est l'idée même de merveilleux qui est en cause.

Néanmoins, l'attrait fort répandu pour les phénomènes extraordinaires manifeste de manière étonnante à quel point la modernité a échoué dans sa tentative de faire table rase du mythos pour glorifier le logos. Tant que la science était elle-même enveloppée d'un discours mythique promettant l'âge d'or pour bientôt, on pouvait se faire illusion. La crise de la modernité a crevé cette bulle de rêve. Si la science et les techniques peuvent encore améliorer la vie humaine, il devient de plus en plus difficile d'attendre d'elles toutes les réponses.

Toutefois, les ressources de la théologie chrétienne permettent de lire la situation présente d'une manière originale. Le rapport aux médiations constitue une première clef de lecture intéressante. La fascination du merveilleux sous toutes ses formes mérite mieux qu'une condamnation a priori parce qu'elle peut manifester une ouverture au mystère. De plus, le principe sacramentel nous empêche de mépriser les médiations par principe, ou de balayer du revers de la main les médiations jugées déplaisantes. C’est dans le cadre de la relecture d'un itinéraire personnel ou communautaire que les médiations prennent sens. Elles n'ont pas de sens " en soi ». La manière dont d'autres personnes construisent du sens à partir d'elles s'avère souvent surprenante. En même temps, toute médiation, même celle que l'on préfère, doit être considérée avec le recul nécessaire pour éviter de finir par y enfermer l'absolu. 
D'autre part, la réflexion chrétienne sur l'espérance et la fatalité, clef de lecture complémentaire, permet de situer l'attrait pour la magie, la divination et le merveilleux sur la ligne de front d'un combat jamais terminé avec ce poids d'amor fati caché dans le secret des cœurs et des consciences. La foi chrétienne, lorsqu'elle est fidèle à l'intuition évangélique, joue le rôle interpellant et dérangeant de ferment d'espérance, de germe de liberté. Cette espérance nourrit le courage d'assumer jusqu'aux pires événements de l'existence, à l'image du crucifié ressuscité, afin qu'ils puissent devenir source de vie et de libération: "Que la force de la liberté qui nous a été donnée par l'Esprit soit toujours plus forte que la condamnation de tous les destins. Et cela, parce que nous, nous sommes destinés à la liberté des enfants de Dieu. ${ }^{17}$

\section{RÉSUMÉ}

La popularité croissante de pratiques et de croyances irrationnelles, en dedans comme en dehors des grandes Églises, lance un défi important à la théologie, celui d'examiner à nouveaux frais les phénomènes habituellement qualifiés de magie et de superstition. Les balises posées par la théologie scolastique dans le domaine du merveilleux ont été remises en question radicalement par le choc de la modernité. Quelles pistes seraient alors susceptibles de renouveler l'interprétation théologique chrétienne de la magie et des phénomènes extraordinaires? L'article en explore deux : le rapport aux médiations dans le christianisme et l'espérance comme résistance à la fatalité.

\section{ABSTRACT}

The continous evolvement of practices and irrational beliefs happening inside and outside traditional churches challenged theology when it came to phenomena qualified as magical and supersitious. The boundaries fixed by scholastic theology, regarding the sensational domain, are questioned by modernity. What are the new ways that can be used to renew christian theological interpretation of magical and extraordinary phenomena? The article propose two ways: the relation to mediations in christianity and hope to resist fatality.

17. Ignacio Madera VARGAS, "Une expérience colombienne de la fatalité : une parole habitée par le destin interpelle une théologie de la liberté » dans Adolphe GESCHÉ (dir.), op. cit., p. 49. 